\title{
L-SERIES AND MODULAR FORMS OF HALF-INTEGRAL WEIGHT
}

\author{
RHONDA L. HATCHER
}

(Communicated by Dennis A. Hejhal)

\begin{abstract}
Let $f$ be a normalized Hecke eigenform of weight $2 k$, with $k$ odd. The main result of this paper is an equation representing the value of $L(f, s) L(f \otimes \varepsilon, s)$ at $s=k$ in terms of the Fourier coefficients of a modular form of half-integral weight.
\end{abstract}

\section{INTRODUCTION}

Let $N$ be a prime, $f \in S_{2 k}^{\text {new }}\left(\Gamma_{0}\right)$. In this paper, we will derive an equation for the value of the product of $L$-series, $L(f, k) L(f \otimes \varepsilon, k)$ at $s=k$ in terms of the squares of the Fourier coefficients of a modular form of half-integral weight. The main identity is a geometric proof of a formula due to Waldspurger [13]. The method of the proof is a generalization of the approach used by Gross [3] in proving the result for $k=1$.

\section{THE DIRICHLET $L$-SERIES}

Let $K$ be an imaginary quadratic field of discriminant $-D$ where $N$ is inert. Let $\mathcal{O}$ be the ring of integers in $K$ and $A=$ ideal class in $K$. Set $\varepsilon=$ quadratic character of $(\mathbb{Z} / D \mathbb{Z})^{*}$ determined by $\varepsilon(p)=\left(\frac{-D}{p}\right)$ for $p \nmid D$ and $2 u=$ number of units in $K$, and set

$$
r_{A}(m)= \begin{cases}\text { number of ideals of norm } m \text { in } A & \text { for } m \geq 1, \\ 1 /(2 u) & \text { for } m=0 .\end{cases}
$$

We now define a Dirichlet series associated with $f$ and the ideal class $A$. Let $\sum_{m \geq 1} a_{m} q^{m}$, with $q=e^{2 \pi i z}$, be the Fourier expansion of $f$. Define the Dirichlet $L$-series associated with $f$ and the ideal class $A$ by

$$
L(f, A, s)=\sum_{\substack{m \geq 1 \\(m, N)=1}}\left(\frac{\varepsilon(m)}{m^{2 s-2 k+1}}\right) \sum_{m \geq 1}\left(\frac{a_{m} r_{A}(m)}{m^{s}}\right) .
$$

$L(f, A, s)$ has an analytic continuation to an entire function of $s$. For proof of this fact see [4].

Received by the editors February 14, 1993.

1991 Mathematics Subject Classification. Primary 11F66; Secondary 11G40.

(C) 1994 American Mathematical Society $0002-9939 / 94 \$ 1.00+\$ .25$ per page 
Let $\chi$ be a complex character of the group $\operatorname{Pic}(\mathscr{O})$, and suppose $f=$ $\sum_{m \geq 1} a_{m} q^{m}$ is a normalized eigenform of weight $2 k$ for the Hecke algebra T. Define

$$
L(f, \chi, s)=\sum_{A} \chi(A) L(f, A, s),
$$

where the sum is over all $A$ in $\operatorname{Pic}(\mathscr{O})$.

It can be shown that if $\varepsilon(N)=1$, then $L(f, \chi, s)$ vanishes at $s=k$. The values of $L(f, \chi, s)$ at $s=k$ in the case $\varepsilon(N)=-1$ were studied in [6]. In particular, an equation representing the value of $L(f, \chi, k)$ in terms of height pairings of special points on a vector bundle $V$ is derived. In the next section we will describe the vector bundle $V$ and the equation for $L(f, \chi, k)$.

\section{The RePresentation of $L(f, \chi, k)$ IN TERMS OF A height Pairing}

Let $B$ be the quaternion algebra over $\mathbb{Q}$ ramified at the rational prime $N$ and at $\infty$. Let $R$ be a maximal order of $B$. Let $\widehat{\mathbb{Z}}=\lim \mathbb{Z} / n \mathbb{Z}=\prod_{p} \mathbb{Z}_{p}$ be the profinite completion of $\mathbb{Z}$, and let $\widehat{\mathbb{Q}}=\widehat{\mathbb{Z}} \otimes \mathbb{Q}=\bigsqcup_{p} \mathbb{Q}_{p}$ be the ring of finite adèles of $\mathbb{Q}$. Set $B_{p}=B \otimes \mathbb{Q}_{p}$. Then $R_{p}=R \otimes \mathbb{Z}_{p}$ is the local component of $R$ in $B_{p}$. Let $\widehat{B}=B \otimes \widehat{\mathbb{Q}}=\bigsqcup_{p} B_{p}$ and $\widehat{R}=R \otimes \widehat{\mathbb{Z}}=\prod_{p} R_{p}$.

Let $B_{0}=\{b \in B: \operatorname{Tr}(b)=0\}$, and let $U$ be the representation of $B^{*}$ on the elements $b_{0} \in B_{0}$ with action by $B^{*}$ defined by $b_{0} \gamma=(1 / \mathbb{N}(\gamma)) \gamma^{-1} b_{0} \gamma$. The center $\mathbb{Q}^{*}$ of $B^{*}$ acts by $b_{0} x=x^{-2} b_{0}$ for $x \in \mathbb{Q}^{*}$. An inner product on $U$ is given by $\left[u_{1}, u_{2}\right]=\frac{1}{2} \operatorname{Tr}\left(u_{1} \overline{u_{2}}\right)$. Let $W_{2 k-1}$ be the inner product space $\operatorname{Sym}^{2 k-2}(W)$, where $W=\mathbb{C} x \oplus \mathbb{C} y$ is the two-dimensional representation of $\mathrm{SU}(2)$ with $[x, x]=[y, y]=1$ and $[x, y]=0$. Since $W_{3}=\operatorname{Sym}^{2}(W) \cong$ $\{b \in B: \operatorname{Tr}(b)=0\}$ and $W_{2 k-1}=\operatorname{Sym}^{2 k-2}(W) \subset \operatorname{Sym}^{k-1}\left(\operatorname{Sym}^{2}(W)\right)$, we can write the inner product space $\operatorname{Sym}^{k-1}(U)$ as the orthogonal direct sum $\operatorname{Sym}^{k-1}(U)=W_{2 k-1} \oplus M$. Note that $W_{2 k-1}$ is the unique irreducible summand of highest weight $2 k-2$, and it is a representation of $B^{*}$ of dimension $2 k-1$.

The quaternion algebra $B$ over $\mathbb{Q}$ corresponds to an algebraic curve $Y$ of genus zero over $\mathbb{Q}$ as described in $[6$, p. 541]. The vector bundle $V$ is defined by

$$
V=\widehat{R}^{*} \backslash \widehat{B}^{*} \times Y \times W_{2 k-1} / B^{*} .
$$

Let $\left\{I_{1}, I_{2}, \ldots, I_{n}\right\}$ be a set of left ideals of $R$ which represent the distinct ideal classes. The set $\left\{I_{1}, I_{2}, \ldots, I_{n}\right\}$ corresponds to a choice of elements $g_{1}, g_{2}, \ldots, g_{n}$ in $\widehat{R}^{*} \backslash \widehat{B}^{*}$ such that $\widehat{B}^{*}=\bigcup_{i=1}^{n} \widehat{R}^{*} g_{i} B^{*}$. Let $R_{i}$ denote the right order of $I_{i}$. For each $\Gamma_{i}=R_{i} /\langle \pm 1\rangle$, define $W^{\Gamma_{i}}=\{w \in W: \gamma(w)=$ $w$ for all $\gamma \in \Gamma_{i}$. Define $\operatorname{Pic}(V)=\bigoplus_{i=1}^{n} W^{\Gamma_{i}}$. Any $v \in V$ is equivalent to the double coset $\left(g_{i}, y, w\right)$ for some $i$. Define the class of the point $v$ to be the projection of $w$ in $W^{\Gamma_{i}}$ given by class $(v)=\frac{1}{\left|\Gamma_{i}\right|} \sum_{\gamma \in \Gamma_{i}} \gamma(w)$.

Define a mapping $\langle$,$\rangle from V \times V$ into $\mathbb{Q}$ as follows. If $v_{1}=g \times \widetilde{y_{1}} \times \widetilde{w_{1}} \cong$ $g_{i} \times y_{1} \times w_{1}$ and $v_{2}=h \times \widetilde{y_{2}} \times \widetilde{w_{2}} \cong g_{j} \times y_{2} \times w_{2}$, then

$$
\left\langle v_{1}, v_{2}\right\rangle= \begin{cases}0 & \text { if } i \neq j, \\ \sum_{\gamma \in \Gamma_{i}}\left[w_{1}, \gamma\left(w_{2}\right)\right]_{W_{2 k-1}} & \text { if } i=j .\end{cases}
$$

This pairing induces a height pairing $($,$\rangle on \operatorname{Pic}(V)$. 
We now define a special element $w_{0}$ of $\operatorname{Sym}^{2 k-2}(W)$. This $w_{0}$ will be used in the definition of a special point of $V$ of discriminant $-D$. For the remainder of the paper, we fix $K$ an imaginary quadratic field of discriminant $-D$, where the prime $N$ is inert, and an embedding $f: K \rightarrow B$. Let $v_{0}=\sqrt{-D} \in U$. Then $v_{0}^{k-1}$ lies in $\operatorname{Sym}^{k-1}(U)$. Let $w_{0} \in \operatorname{Sym}^{2 k-2}(W)$ be the component of $v_{0}^{k-1}$ in $\operatorname{Sym}^{2 k-2}(W)$.

Let $X=\widehat{R}^{*} \backslash \widehat{B}^{*} \times Y / B^{*}$. There exists a canonical identification $Y(K) \simeq$ $\operatorname{Hom}(K, B)$ as described in [6, p. 541]. The special points of $V$ of discriminant $-D$ are defined to be points of the form $v=g \times y \times w_{0}$, where $g \times y$ lies in the image of $\widehat{R}^{*} \backslash \widehat{B}^{*} \times Y(K)$ in $X(K)$, and the embedding $f$ corresponding to $y$ satisfies $f(K) \cap g^{-1} \widehat{R} g=f\left(\mathscr{O}_{-D}\right)$, where $\mathscr{O}_{-D}$ is the order of discriminant $-D$. An action of $\operatorname{Pic}(\mathscr{O})$ on the special points of $V$ of discriminant $-D$ given by $v \rightarrow v_{A}$ for $A \in \operatorname{Pic}(\mathscr{O})$ is defined in [6, p. 545].

Define $e_{\chi}=\sum_{A} \chi^{-1}(A) v_{A}$, where the sum is over all $A$ in $\operatorname{Pic}(\mathscr{O})$. Let $e_{f, \chi}$ be the projection of the divisor $e_{\chi}$ in $\operatorname{Pic}(V) \otimes \mathbb{C}$ to the $f$-isotypical eigenspace for the Hecke algebra $\mathbb{T}$. The following result is proved in [6, Proposition 8.2 , p. 558] for the case $D$ prime, and it follows from [7, Proposition 4.1, p. 340] that the result can be extended to the case $D$ composite.

Proposition 1. If $\varepsilon(N)=-1$, then

$$
L(f, \chi, k)=\frac{(f, f)}{u^{2} D^{k-\frac{1}{2}}(k-1) !^{2}}\left\langle e_{f, \chi}, e_{f, \chi}\right\rangle,
$$

where $(, \quad)$ is the Petersson product as defined in [6, p. 547].

In the special case $\chi=1, L(f, \chi, k)$ can be written in the form

$$
L(f, \chi, k)=L(f, k) L(f \otimes \varepsilon, k),
$$

where $f \otimes \varepsilon=\sum_{m \geq 1} a_{m} \varepsilon(m) q^{m}$ is the twist of $f, L(f, s)=\sum_{m \geq 1} a_{m} m^{-s}$, and $L(f \otimes \varepsilon, s)=\sum_{m \geq 1} a_{m} \varepsilon(m) m^{-s}$.

Define $e_{D}$ to be the class in $\operatorname{Pic}(V)$ of the rational divisor

$$
\frac{1}{2 u} \sum_{\operatorname{disc}(v)=-D}(v) \text {. }
$$

Then, as explained in $[6$, p. 560], in the special case $\chi=1$ Proposition 1 becomes

Corollary 1. If $\varepsilon(N)=-1$, then

$$
L(f, k) L(f \otimes \varepsilon, k)=\frac{(f, f)}{D^{k-\frac{1}{2}}(k-1) !^{2}}\left\langle e_{f, D}, e_{f, D}\right\rangle .
$$

In the next section we will use the result of Corollary 1 to arrive at an equation representing the value of $L(f, k) L(f \otimes \varepsilon, k)$ in terms of the Fourier coefficients of a modular form of half-integral weight.

\section{THE MAIN IDENTITY}

Define the formal series $g=\sum_{D>1} e_{D} q^{D}$, with the $e_{D}$ as defined above. We will need the following result. 
Lemma 1. For all $e \in \operatorname{Pic}(V)$ the series

$$
g(e)=\sum_{D>1}\left\langle e, e_{D}\right\rangle q^{D}
$$

is a modular form of weight $k+\frac{1}{2}$ on $\Gamma_{0}(4 N)$ with integral coefficients.

Proof. Let $\left\{\alpha_{j}\right\}$ be the basis for $\operatorname{Pic}(V)$ that you get by tensoring the basis $\left\{e_{1}, e_{2}, \ldots, e_{n}\right\}$ of $\operatorname{Pic}(X)$ described in [3] with the basis of $\operatorname{Sym}^{2 k-2}(W)$ given by $\left\{x^{2 k-2}, x^{2 k-3} y, \ldots, y^{2 k-2}\right\}$. Recall that $\operatorname{Sym}^{2 k-2}(W)$ embeds into $\operatorname{Sym}^{k-1}(U)$ as described earlier. It will suffice to show that for every $\alpha_{j}, g\left(\alpha_{j}\right)$ is a modular form of weight $k+\frac{1}{2}$ on $\Gamma_{0}(4 N)$.

Suppose $\alpha_{j}=e_{i} \otimes x^{l} y^{m}$. Then

$$
g\left(\alpha_{j}\right)=g\left(e_{i} \otimes x^{l} y^{m}\right)=\sum_{D>1}\left\langle e_{i} \otimes x^{l} y^{m}, e_{D}\right\rangle
$$

Using the fact that

$$
e_{D}=\sum_{i=1}^{n} a_{i}(D)\left(e_{i}^{\vee} \otimes w_{0}\right)
$$

where $w_{0}$ is the component of $(\sqrt{-D})^{k-1}$ in $\operatorname{Sym}^{2 k-2}(W), e_{i}^{\vee}=e_{i} /\left|\Gamma_{i}\right|$, and $a_{i}(D)$ is the number of embeddings of $\mathscr{O}_{D} \rightarrow R_{i}$ modulo $R_{i}^{*}$, it follows that

$$
g\left(\alpha_{j}\right)=\sum_{D>1}\left\langle e_{i} \otimes x^{l} y^{m}, \sum_{i=1}^{n} a_{i}(D)\left(e_{i}^{\vee} \otimes w_{0}\right)\right\rangle q^{D}=\sum_{D>1} a_{i}(D)\left[x^{l} y^{m}, w_{0}\right] q^{D} \text {. }
$$

Applying a result of Pizer [11, Proposition 2.10], we see that the inner product $\left[x^{l} y^{m}, w_{0}\right]$ is equal to $p(\sqrt{-D})$, where $p$ is a spherical polynomial of degree $k-1$ in three variables $x_{1}, x_{2}, x_{3}$, with $\sqrt{-D}=x_{1} \mu_{1}+x_{2} \mu_{2}+x_{3} \mu_{3}$, where $\left\{\mu_{1}, \mu_{2}, \mu_{3}\right\}$ is a basis of $U$. Following the work of Gross in [3], we let $S_{i}$ be the suborder of index 8 in $R_{i}$ which is defined by $S_{i}=\mathbb{Z}+2 R_{i}$, and let $S_{i}^{0}$ be the subgroup of elements of trace zero in $S_{i}$. Then $S_{i}^{0}$ has rank three over $\mathbb{Z}$. From [3], we know that $a_{i}(D)$ is one half of the number of elements $b \in R_{i}$ with $b \equiv 0,1 \bmod 2 R_{i}, \operatorname{Tr} b=0$, and $\mathbb{N} b=D=-b^{2}$. It follows that

$$
g\left(\alpha_{j}\right)=\sum_{D>1} a_{i}(D) p(\sqrt{-D}) q^{D}=\frac{1}{2} \sum_{b \in S_{i}^{o}} p(b) q^{N b} .
$$

Since $\mathbb{N} b$ is a positive definite ternary quadratic form and $p(b)$ is a spherical polynomial of degree $k-1$ in three variables, it follows from a result of Schoeneberg [12] generalized by Pfetzer [10, p. 452] that $g\left(\alpha_{i}\right)$ is a modular form of weight $k+\frac{1}{2}$ on $\Gamma_{0}(4 N)$. The lemma now follows.

Define $e_{f}$ to be a nonzero element in the $f$-isotypical component of $\operatorname{Pic}(V) \otimes$ $\mathbb{R}$, where $f$ is an eigenform for the Hecke algebra $\mathbb{T}$. Then from Lemma 1 , we know that

$$
g\left(e_{f}\right)=\sum_{D}\left\langle e_{f}, e_{D}\right\rangle q^{D} \equiv \sum_{D} m_{D} q^{D}
$$

is a modular form of weight $k+\frac{1}{2}$ on $\Gamma_{0}(4 N)$ with integral coefficients. Furthermore, $g\left(e_{f}\right)$ is an eigenform for the Hecke algebra on the space of modular 
forms of weight $k+\frac{1}{2}$ on $\Gamma_{0}(4 N)$ with eigenvalues corresponding to the eigenvalues of $f$. This fact follows from an argument analogous to that given by Gross in [3] for the case $k=1$.

The main result, which we prove next, gives a representation of the value of $L(f, k) L(f \otimes \varepsilon, k)$ in terms of the Fourier coefficients $m_{D}$. The formula in Proposition 2 is due to Waldspurger [13].

Proposition 2. If $\varepsilon(N)=-1$, then

$$
L(f, k) L(f \otimes \varepsilon, k)=\frac{(f, f)}{D^{k-\frac{1}{2}(k-1) ! 2}} \frac{m_{D}^{2}}{\left\langle e_{f}, e_{f}\right\rangle} .
$$

Proof. From Proposition 1, we know that

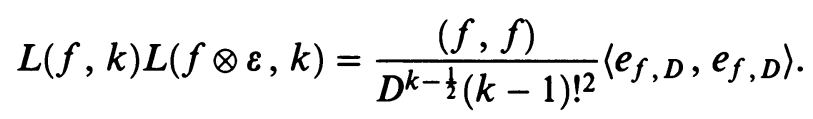

Hence, it will suffice to show that

$$
\left\langle e_{f, D}, e_{f, D}\right\rangle=\frac{m_{D}^{2}}{\left\langle e_{f}, e_{f}\right\rangle} .
$$

To see that this is true, first note that $m_{D}=\left\langle e_{f}, e_{D}\right\rangle=\left\langle e_{f}, e_{f, D}\right\rangle$. Therefore,

$$
e_{f, D}=\frac{m_{D}}{\left\langle e_{f}, e_{f}\right\rangle} e_{f}
$$

in $(\operatorname{Pic}(V) \otimes \mathbb{R})^{f}$. It follows that

$$
\begin{aligned}
\left\langle e_{f, D}, e_{f, D}\right\rangle & =\left\langle\frac{m_{D}}{\left\langle e_{f}, e_{f}\right\rangle} e_{f}, \frac{m_{D}}{\left\langle e_{f}, e_{f}\right\rangle} e_{f}\right\rangle \\
& =\frac{m_{D}^{2}}{\left\langle e_{f}, e_{f}\right\rangle^{2}}\left\langle e_{f}, e_{f}\right\rangle=\frac{m_{D}^{2}}{\left\langle e_{f}, e_{f}\right\rangle} .
\end{aligned}
$$

This completes the proof.

\section{REFERENCES}

1. S. Böcherer and R. Schulze-Pillot, On a theorem of Waldspurger and on Eisenstein series of Klingen type, Math. Ann. 288 (1990), 361-388.

2. __ The Dirichlet series of Koecher and Maas and modular forms of weight 3/2, preprint.

3. B. Gross, Heights and the special values of L-series, CMS Conf. Proc., vol. 7, Amer. Math. Soc., Providence, RI, 1987, pp. 115-187.

4. B. Gross and D. Zagier, Heegner points and derivatives of L-series, Invent. Math. 84 (1986), 225-320.

5. B. Gross, W. Kohnen, and D. Zagier, Heegner points and derivatives of L-series. II, Math. Ann. 278 (1987), 497-562.

6. R. Hatcher, Heights and L-series, Canad. J. Math 42 (1990), 533-560.

7. __ Special values of L-series, Proc. Amer. Math. Soc. 114 (1992), 337-343.

8. W. Kohnen, Newforms of half-integral weight, J. Reine Angew. Math. 333 (1982), 32-72.

9. $\ldots$, Fourier coefficients of modular forms of half-integral weight, Math. Ann. 271 (1985), 237-268.

10. V. Pfetzer, Die Wirkung der Modulsubstitutionen auf mehrfache Thetareihen zu quadratischen Formen ungerader Variablenzahl, Arch. Math. 4 (1953), 448-454. 
11. A. Pizer, An algorithm for computing modular forms on $\Gamma_{0}(N)$, J. Algebra 64 (1980), 340-390.

12. B. Schoeneberg, Das Verhalten von mehrfachen Thetareihen bei Modulsubstitutionen, Math. Ann. 116 (1939), 511-523.

13. J.-L. Waldspurger, Sur les coefficients de Fourier des formes modulaires de poids demi-entier, J. Math. Pures Appl. (9) 60 (1981), 375-484.

Department of Mathematics, Texas Christian University, Fort Worth, Texas 76129

E-mail address: hatcheregamma.is.tcu.edu 